\title{
ON THE DISORDER PROBLEM FOR A NEGATIVE BINOMIAL PROCESS
}

\author{
BRUNO BUONAGUIDI $* * *$ AND \\ PIETRO MULIERE, ${ }^{* * * *}$ Bocconi University
}

\begin{abstract}
We study the Bayesian disorder problem for a negative binomial process. The aim is to determine a stopping time which is as close as possible to the random and unknown moment at which a sequentially observed negative binomial process changes the value of its characterizing parameter $p \in(0,1)$. The solution to this problem is explicitly derived through the reduction of the original optimal stopping problem to an integrodifferential free-boundary problem. A careful analysis of the free-boundary equation and of the probabilistic nature of the boundary point allows us to specify when the smooth fit principle holds and when it breaks down in favour of the continuous fit principle.
\end{abstract}

Keywords: Disorder problem; free-boundary problem; negative binomial process; optimal stopping; principles of continuous and smooth fit; regular boundary; sequential detection

2010 Mathematics Subject Classification: Primary 60G40

Secondary 62C10; 62L10; 35R35

\section{Introduction}

In this paper we study the Bayesian disorder problem for a negative binomial process. Let us recall that a negative binomial process $X=\left(X_{t}\right)_{t \geq 0}$ with parameter $p \in(0,1)$ is a Lévy process whose characteristic function $\hat{u}_{t}(z):=\mathbb{E}\left[\mathrm{e}^{\mathrm{i} z X_{t}}\right]$ is

$$
\hat{u}_{t}(z)=\exp \left\{t \int_{(0,+\infty)}\left(\mathrm{e}^{\mathrm{i} z x}-1\right) v(\mathrm{~d} x)\right\}=\left(\frac{p}{1-q \mathrm{e}^{\mathrm{i} z}}\right)^{t}, \quad z \in \mathbb{R},
$$

where $q:=1-p$ and $v(\{x\}):=q^{x} / x, x=1,2, \ldots$, is the so-called Lévy measure. We easily deduce that for any $t>0, X_{t}$ follows a negative binomial distribution with parameters $t$ and $p \in(0,1)$ or, equivalently, that $X$ is a compound Poisson process with intensity $-\log p$ (i.e. the time between two successive jumps has exponential distribution with mean $1 / \log (1 / p))$ and jumps whose magnitude follows the logarithmic distribution $\zeta(\{x\})=-q^{x} /(x \log p)$, $x=1,2, \ldots$.

Our problem can be stated as follows: at time $t=0$ we begin to observe continuously a negative binomial process $X=\left(X_{t}\right)_{t \geq 0}$; at a random and unobservable time $\vartheta$, known as disorder time or change point, the characterizing parameter $p$ of $X$ shifts from $p_{0}$ to $p_{1}$. It is assumed that $\vartheta=0$, with probability $\pi$, and, given that it is greater than 0 , is exponentially distributed with parameter $\lambda>0$. The disorder problem for a negative binomial process aims to determine a stopping time at which one can declare that the disorder has occurred. This stopping time must be as close as possible to $\vartheta$ in the sense that the sum between the probability of a

Received 3 December 2013; revision received 2 March 2014.

* Postal address: Department of Decision Sciences, Bocconi University, Via Roentgen 1, 20136 Milan, Italy.

** Email address: bruno.buonaguidi@phd.unibocconi.it

*** Email address: pietro.muliere@unibocconi.it 
false alarm and the expected cost due to the delay for a correct identification of the disorder time is minimized.

Explicit solutions to the quickest detection of a change in the probability characteristics of the ongoing process were obtained by Shiryaev (1978, Section 4.4), for the drift of a Brownian motion, Peskir and Shiryaev (2002), for the intensity of a Poisson process, and Gapeev (2005), for a compound Poisson process whose intensity equals the mean of the exponential distribution of its jumps. The method of proof that these works are based on consists of reducing the initial optimal stopping problem to a free-boundary problem, and specifying when the smooth fit principle holds at the optimal boundary point and when it breaks down in favour of the continuous fit principle.

Our study adopts the same free-boundary approach. In particular, after a formal description of the problem in Section 2, in Section 3 we reduce the original optimal stopping problem to a free-boundary problem for an integro-differential operator. In Section 4 we analyze the regularity of the optimal boundary point. The existence of a singularity point in the freeboundary equation and the results of Section 4 allow us to specify when the smooth fit holds and when it is replaced by the continuous fit principle. In Section 5 we state and prove the main theorem of the paper and in Section 6 we conclude with a summary and discussion.

We underline that although the compound Poisson representation of a negative binomial process makes possible the application of the numerical scheme developed by Dayanik and Sezer (2006), we provide the next example of a process whose disorder problem admits a closed form solution. The exponential distribution of the change point and the linear penalty for the correct identification of the disorder that we assume throughout this paper ensure an explicit and analytical tractability of the problem, which, instead, would require numerical solution methods if these settings were removed (see, e.g. Bayraktar and Sezer (2009) and Dayanik (2010)). Furthermore, from (1.1) we observe that the height of the jumps of a negative binomial process ranges in the positive integer numbers and $\mathbb{E}\left[X_{t}\right]=q t / p<q t / p^{2}=\operatorname{var}\left[X_{t}\right], t>0$. These facts make our results valid in those situations where the Poisson process is inappropriate, namely, when the events occur in clusters and are characterized by overdispersion. Our study is motivated by the application of the negative binomial process and the related negative binomial distribution in several fields, such as distribution theory (Anscombe (1950), Barndorff-Nielsen and Yeo (1969), Vaillant (1991), Zhou and Carin (2013)), agriculture and pest management (Mukhopadhyay (2014) and Mukhopadhyay and de Silva (2005)), cosmology (Carruthers and Minh (1983)), entomology (Nedelman (1983) and Wilson and Room (1983)), and hydrology (Kozubowski and Podgórski (2009)). Problems of sequential testing for such a process were faced in Buonaguidi and Muliere (2013a) and Buonaguidi and Muliere (2013b).

\section{Formulation of the problem}

On the statistical space $\left(\Omega, \mathcal{F}, \mathbb{P}_{\pi}\right)$ the unobservable random variable $\vartheta$ is defined; the probability measure $\mathbb{P}_{\pi}$ is such that $\mathbb{P}_{\pi}(\vartheta=0)=\pi$ and $\mathbb{P}_{\pi}(\vartheta>t \mid \vartheta>0)=\mathrm{e}^{-\lambda t}$, for $t \geq 0$, where $\lambda>0$ and $\pi \in[0,1]$ are given. The same space hosts two independent and unobservable negative binomial processes $X^{0}=\left(X_{t}^{0}\right)_{t \geq 0}$ and $X^{1}=\left(X_{t}^{1}\right)_{t \geq 0}$, with known parameters $p_{0}, p_{1} \in(0,1), p_{0} \neq p_{1}$, respectively. They are assumed to be independent of $\vartheta$. The process $X=\left(X_{t}\right)_{t \geq 0}$ we observe is given by

$$
X_{t}:=\int_{0}^{t}\left(1-\vartheta_{s^{-}}\right) \mathrm{d} X_{s}^{0}+\int_{0}^{t} \vartheta_{s^{-}} \mathrm{d} X_{s}^{1},
$$

where $\vartheta_{s}:=\mathbf{1}_{\{\vartheta \leq s\}}$, for all $t, s \geq 0$ and $\mathbf{1}$ is the indicator function. 
Let $\mathcal{F}^{X}=\left(\mathcal{F}_{t}^{X}\right)_{t \geq 0}$, with $\mathcal{F}_{t}^{X}:=\sigma\left\{X_{s}: 0 \leq s \leq t\right\}$, be the natural filtration of $X$. The Bayesian disorder problem for a negative binomial process aims at computing

$$
V(\pi):=\inf _{\tau}\left(\mathbb{P}_{\pi}(\tau<\vartheta)+c \mathbb{E}_{\pi}[\tau-\vartheta]^{+}\right), \quad c>0,
$$

and finding, among all the $\mathcal{F}^{X}$ stopping times, the optimal stopping time at which the expression in the brackets on the right-hand side of (2.2) reaches the infimum. It is easily seen that introducing the posterior probability process $\left(\pi_{t}\right)_{t \geq 0}$, being $\pi_{t}:=\mathbb{P}_{\pi}\left(\vartheta \leq t \mid \mathcal{F}_{t}^{X}\right)$ with $\pi_{0}=\pi, V(\pi)$ can be equivalently written as

$$
V(\pi)=\inf _{\tau} \mathbb{E}_{\pi}\left[1-\pi_{\tau}+c \int_{0}^{\tau} \pi_{t} \mathrm{~d} t\right],
$$

where the infimum is taken over all the stopping times with respect to the natural filtration of $\left(\pi_{t}\right)_{t \geq 0}$, which, as shown by (2.5) below, coincides with $\mathcal{F}^{X}$. It is well known (see, e.g. Shiryaev (1978, pp. 197-198)) that the map $\pi \mapsto V(\pi)$ is decreasing and concave on $[0,1]$ and that

$$
\tau_{\pi}^{\star}:=\inf \left\{t \geq 0: \pi_{t} \geq B^{\star}, \pi_{0}=\pi\right\}
$$

is optimal in (2.3), where $B^{\star}$ is the smallest number $\pi \in[0,1]$ satisfying $V(\pi)=1-\pi$.

The value function $V(\pi)$ and the threshold $B^{\star}$ can be obtained as a solution of a suitable free-boundary problem, involving the infinitesimal operator $\mathbb{L}$ of $\left(\pi_{t}\right)_{t \geq 0}$. In order to derive its expression, we follow the analogous schema of arguments of Peskir and Shiryaev (2002). Using standard means (see, e.g. Sato (1999, Theorem 33.2, p. 219)), we can show that the likelihood ratio process $\varphi_{t}:=\pi_{t} /\left(1-\pi_{t}\right), t \geq 0$, is

$$
\begin{aligned}
\varphi_{t}= & \exp \left(\log \left(\frac{q_{1}}{q_{0}}\right) X_{t}+\left(\lambda-\log \left(\frac{p_{0}}{p_{1}}\right)\right) t\right) \\
& \times\left(\varphi_{0}+\int_{0}^{t} \lambda \exp \left(-\log \left(\frac{q_{1}}{q_{0}}\right) X_{s}-\left(\lambda-\log \left(\frac{p_{0}}{p_{1}}\right)\right) s\right) \mathrm{d} s\right),
\end{aligned}
$$

where $q_{i}:=1-p_{i}, i=0,1$. A simple application of Itô's equation and the fact that $\pi_{t}=\varphi_{t} /\left(1+\varphi_{t}\right), t \geq 0$, imply that $\left(\varphi_{t}\right)_{t \geq 0}$ and $\left(\pi_{t}\right)_{t \geq 0}$ satisfy

$$
\begin{aligned}
\mathrm{d} \varphi_{t} & =\left(\lambda\left(1+\varphi_{t}\right)-\log \left(\frac{p_{0}}{p_{1}}\right) \varphi_{t}\right) \mathrm{d} t+\varphi_{t^{-}} \sum_{x=1}^{\infty}(\xi(x)-1) \mu^{X}(\{x\}, \mathrm{d} t), \\
\mathrm{d} \pi_{t} & =\lambda\left(1-\pi_{t}\right) \mathrm{d} t+\sum_{x=1}^{\infty} \frac{\pi_{t^{-}}\left(1-\pi_{t^{-}}\right)(\xi(x)-1)}{1+\pi_{t^{-}}(\xi(x)-1)}\left(\mu^{X}-v^{X}\right)(\{x\}, \mathrm{d} t),
\end{aligned}
$$

being $\xi(x):=\left(q_{1} / q_{0}\right)^{x}, \mu^{X}(\{x\},(0, t])$ the number of jumps of $X$ of magnitude $x$ in the time interval $(0, t]$, and $v^{X}(\{x\}, \mathrm{d} t):=\mathrm{d} t\left(\pi_{t^{-}} q_{1}^{x}+\left(1-\pi_{t^{-}}\right) q_{0}^{x}\right) / x$ its compensator. By Itô's equation, the infinitesimal operator $\mathbb{L}$ of $\left(\pi_{t}\right)_{t \geq 0}$ acts on a function $f \in C^{1}[0,1]$ such that

$$
\begin{aligned}
(\mathbb{L} f)(\pi)= & \left(\lambda-\log \left(\frac{p_{0}}{p_{1}}\right) \pi\right)(1-\pi) f^{\prime}(\pi)+f(\pi)\left((1-\pi) \log p_{0}+\pi \log p_{1}\right) \\
& +\sum_{x=1}^{\infty} f\left(\frac{\pi q_{1}^{x}}{\pi q_{1}^{x}+(1-\pi) q_{0}^{x}}\right) \frac{\left(\pi q_{1}^{x}+(1-\pi) q_{0}^{x}\right)}{x},
\end{aligned}
$$

that for $\lambda=0$ reduces to the analogous expression given in Buonaguidi and Muliere (2013a, Equation 6.3). 


\section{The free-boundary problem}

The strong Markov property of $\left(\pi_{t}\right)_{t \geq 0}$, evident from (2.6), and the general theory of optimal stopping (see, e.g. Peskir and Shiryaev (2006, Chapters 3 and 4) or Shiryaev (1978, Chapter 3)) naturally entail the formulation of the following free-boundary problem for the unknown map $\pi \mapsto V(\pi)$ and boundary $B^{\star}$ :

$$
\begin{gathered}
(\mathbb{L} V)(\pi)=-c \pi, \quad 0<\pi<B^{\star}, \\
V(\pi)=1-\pi, \quad B^{\star} \leq \pi \leq 1, \\
V\left(B^{\star}-\right)=1-B^{\star}(\text { continuous } f i t), \\
V^{\prime}(0+)=0 \text { (normal entrance). }
\end{gathered}
$$

It is easy to check that the last condition is satisfied by any function solving (3.1) and will prove to be useful when $p_{0}<p_{1}$. Furthermore, in some cases, $V$ and $B^{\star}$ also satisfy

$$
V^{\prime}\left(B^{\star}\right)=-1 \text { (smooth fit). }
$$

Before deriving the solution of the above free-boundary problem, we observe that the 'step' function

$$
S(\pi ; x):=\frac{\pi q_{1}^{x}}{\pi q_{1}^{x}+(1-\pi) q_{0}^{x}}, \quad x=0,1,2, \ldots,
$$

appearing in (2.7), enjoys the following properties:

$$
\begin{gathered}
\frac{\partial S(\pi ; x)}{\partial \pi}>0 ; \\
S(\pi ; x+1)=S(S(\pi ; x) ; 1) ; \\
S(\pi ; x+1)\left\{\begin{array}{ll}
>S(\pi, x), & p_{0}>p_{1}, \\
<S(\pi ; x), & p_{0}<p_{1},
\end{array} \quad x=0,1,2, \ldots ;\right. \\
\lim _{x \rightarrow \infty} S(\pi, x)= \begin{cases}1, & p_{0}>p_{1}, \\
0, & p_{0}<p_{1} .\end{cases}
\end{gathered}
$$

For further reference, we define the 'distance' function

$$
d(\pi ; y):=1+\left\lfloor\frac{\log ((y /(1-y))((1-\pi) / \pi))}{\log \left(q_{1} / q_{0}\right)}\right\rfloor,
$$

where $y$ is a fixed number in $(0,1)$ and $\lfloor x\rfloor$ is the largest integer less than or equal to $x$.

Let us first assume that $p_{0}>p_{1}$. For a fixed $B \in(0,1)$, determine the sequence of points $\cdots<B_{n}<\cdots<B_{1}<B_{0}=: B$ such that $S\left(B_{n} ; 1\right)=B_{n-1}, n \geq 1$. Straightforwardly,

$$
B_{n}=\frac{q_{0}^{n} B}{q_{0}^{n} B+q_{1}^{n}(1-B)} .
$$

Denote by $I_{n}:=\left(B_{n}, B_{n-1}\right], n \geq 1$, and note that, for all $\pi \in(0, B]$,

$$
\pi \in I_{n} \Longleftrightarrow d(\pi ; B)=n \text {. }
$$


Consider (3.1) on $I_{1}$; because of the properties (3.6), (3.7), and (3.8), $S(\pi ; x), x \geq 1$, ranges in $(B, 1]$ so that, according to (3.2), we set $V(S(\pi ; x))=1-S(\pi ; x)$ for $x \geq 1$. In this way we have a first-order linear differential equation, whose unique solution $\pi \mapsto V(\pi ; B)$ on $I_{1}$ is given by

where

$$
V(\pi ; B)=c_{1}(B) V_{g}(\pi)+V_{p, 1}(\pi ; B), \quad \pi \in I_{1},
$$

$$
V_{g}(\pi):= \begin{cases}\frac{(1-\pi)^{\gamma_{1}}}{\left|\lambda-\log \left(p_{0} / p_{1}\right) \pi\right|^{\gamma_{0}}}, & \lambda \neq \log \left(p_{0} / p_{1}\right), \\ (1-\pi) \exp \left(\frac{-\log \left(p_{1}\right)}{\log \left(p_{0} / p_{1}\right)(1-\pi)}\right), & \lambda=\log \left(p_{0} / p_{1}\right)\end{cases}
$$

is a general solution of the homogeneous equation in (3.1), with

and

$$
\gamma_{0}:=\frac{\lambda-\log p_{0}}{\log \left(p_{0} / p_{1}\right)-\lambda}, \quad \gamma_{1}:=-\frac{\log p_{1}}{\log \left(p_{0} / p_{1}\right)-\lambda},
$$

$$
V_{p, 1}(\pi ; B):=\frac{\log p_{0}\left(c+\log p_{1}\right)}{\log p_{1}\left(\lambda-\log p_{0}\right)} \pi-\frac{\log p_{0} \log p_{1}+c \lambda}{\log p_{1}\left(\lambda-\log p_{0}\right)}
$$

is a bounded particular solution of the nonhomogeneous equation in (3.1). The constant term $c_{1}(B)$ is determined by imposing the continuity condition (3.3) at $B$ and is given by

$$
c_{1}(B)=-\frac{1}{V_{g}(B)}\left(\frac{\lambda \log p_{1}+c \log p_{0}}{\log p_{1}\left(\lambda-\log p_{0}\right)} B-\frac{\lambda\left(\log p_{1}+c\right)}{\log p_{1}\left(\lambda-\log p_{0}\right)}\right) .
$$

Moving now onto the generic interval $I_{n}, n \geq 1$; because of the properties (3.6)-(3.8) and by construction of $B_{n}, B_{n-1}, \ldots, B_{1}, S(\pi ; 1) \in I_{n-1}, S(\pi ; 2) \in I_{n-2}, \ldots, S(\pi ; n-1) \in I_{1}$, and $S(\pi ; x) \in(B, 1]$ for $x \geq n$. Thus, setting $V(\pi)$ equal to the solution found on $I_{n-i}$ for $\pi \in I_{n-i}, i=1, \ldots, n-1$, and, according to (3.2), $V(\pi)=1-\pi$ for $\pi \in(B, 1]$, we obtain a first-order linear differential equation that has a unique solution $\pi \mapsto V(\pi ; B)$ on $I_{n}$ given by

$$
V(\pi ; B)=c_{n}(B) V_{g}(\pi)+V_{p, n}(\pi ; B), \quad \pi \in I_{n},
$$

where $\pi \mapsto V_{g}(\pi)$ is a general solution expressed by (3.13), $\pi \mapsto V_{p, n}(\pi ; B)$ is a bounded particular solution and the constant term $c_{n}(B)$ is obtained by imposing a continuity condition on $I_{n} \cup I_{n-1}$ at $B_{n-1}$. Unless $\lambda=0$ as in Buonaguidi and Muliere (2013a, Section 6), $c_{n}(B)$ and $V_{p, n}(\pi ; B)$ for $n \geq 2$ cannot be expressed in terms of elementary functions and we therefore omit their equations.

The general solution of (3.1)-(3.3) is, therefore, given by

$$
V(\pi)= \begin{cases}V(\pi ; B), & 0<\pi<B, \\ 1-\pi, & B \leq \pi \leq 1,\end{cases}
$$

where, by using the distance function (3.9),

$$
V(\pi ; B)=c_{d(\pi ; B)}(B) V_{g}(\pi)+V_{p, d(\pi ; B)}(\pi ; B), \quad \pi \in(0, B] .
$$

Remark 3.1. When $\lambda<\log \left(p_{0} / p_{1}\right)$, the point

$$
\hat{B}:=\frac{\lambda}{\log \left(p_{0} / p_{1}\right)}
$$

is a singularity point of the free-boundary equation (3.1). This is easily observed through (3.13), where $V_{g}(\pi) \rightarrow \infty$ as $\pi \rightarrow \hat{B}$. 
Remark 3.2. If we fix $B=\hat{B}$ from (3.19), according to (3.15) and the previous remark, we see that $V(\pi ; \hat{B})=V_{p, 1}(\pi ; \hat{B})$ for $\pi \in I_{1}$. The unique point satisfying the continuous fit (3.3) is, therefore, $\lambda\left(\log p_{1}+c\right) /\left(\lambda \log p_{1}+c \log p_{0}\right)$, which is equal to $\hat{B}$ only if $c=\log \left(p_{0} / p_{1}\right)-\lambda$. So, for $B=\hat{B}$ and $c \neq \log \left(p_{0} / p_{1}\right)-\lambda$, the free-boundary problem (3.1)-(3.3) has no solution. However, this analytic fact has no relevance for the solution of the optimal stopping problem (2.2)-(2.3).

Remark 3.3. The expressions (3.12)-(3.15) and some simple calculations demonstrate that $\partial V(\pi ; B) / \partial \pi$ is not defined at $\pi=\hat{B}$ (of course, when $B \geq \hat{B}$ ) and that the unique point satisfying the smooth fit (3.5) is

$$
\widetilde{B}:=\frac{\lambda}{\lambda+c} .
$$

These facts imply that $c=\log \left(p_{0} / p_{1}\right)-\lambda$ (for which $\widetilde{B}=\hat{B}$ ) is a breakage condition of the smooth fit principle (3.5); in particular, since $V(\pi ; \hat{B})=V_{p, 1}(\pi ; \hat{B})$ for $\pi \in I_{1}$, we have

$$
\left.\frac{\partial V(\pi ; \hat{B})}{\partial \pi}\right|_{\pi=\hat{B}-}=-\frac{\log p_{0}}{\log p_{1}} \neq-1 .
$$

It will be proved that $\widetilde{B}$ coincides with the optimal stopping boundary $B^{\star}$ from (2.4) whenever $c \geq \log \left(p_{0} / p_{1}\right)-\lambda$.

Let us now assume that $p_{0}<p_{1}$. For a sufficiently small $\varepsilon>0$, according to (3.7), we determine the sequence of points $\varepsilon=: \varepsilon_{0}<\varepsilon_{1}<\cdots<\varepsilon_{n}<\cdots$, such that $S\left(\varepsilon_{n} ; 1\right)=\varepsilon_{n-1}$, $n \geq 1$. Then, defining $I_{n}:=\left[\varepsilon_{n-1}, \varepsilon_{n}\right), n \geq 1$, we observe that $\varepsilon_{n}$ is given by (3.10) and that (3.11) holds, once $B$ is replaced by $\varepsilon$ in the corresponding expressions. Consider (3.1) on $I_{1}$ and observe from (3.6), (3.7), and (3.8) that $S(\pi ; x) \in[0, \varepsilon)$, for $x \geq 1$. According to the normal entrance condition (3.4), we set $V(\pi)=v$ for $\pi \in[0, \varepsilon)$, where $v$ is a given number in $(0,1)$. In this way, we obtain a first-order linear differential equation whose unique solution $\pi \mapsto V(\pi ; \varepsilon, v)$ on $I_{1}$ is

$$
V(\pi ; \varepsilon, v)=c_{1}(\varepsilon) V_{g}(\pi)+V_{p, 1}(\pi ; \varepsilon, v), \quad \pi \in I_{1},
$$

where $\pi \mapsto V_{g}(\pi)$ is a general solution given by (3.13), $\pi \mapsto V_{p, 1}(\pi ; \varepsilon, v)$ is a particular solution given by

$$
V_{p, 1}(\pi ; \varepsilon, v):=\frac{c \log p_{0}}{\log p_{1}\left(\lambda-\log p_{0}\right)} \pi-\frac{c \lambda}{\log p_{1}\left(\lambda-\log p_{0}\right)}+v,
$$

and the constant term $c_{1}(\varepsilon)$, given by

$$
c_{1}(\varepsilon)=-\frac{1}{V_{g}(\varepsilon)}\left(\frac{c \log p_{0}}{\log p_{1}\left(\lambda-\log p_{0}\right)} \varepsilon-\frac{c \lambda}{\log p_{1}\left(\lambda-\log p_{0}\right)}\right),
$$

is obtained by imposing the continuity condition $V(\varepsilon)=v$. Using the same reasoning as in the case of $p_{0}>p_{1}$ when we consider (3.1) on $I_{n}, n \geq 1$, because of (3.6)-(3.8), we note that $S(\pi ; x) \in I_{n-x}, x=1, \ldots, n-1$, and $S(\pi ; x) \in[0, \varepsilon)$ for $x \geq n$. Hence, setting $V(\pi), \pi \in I_{n-i}$, equal to the solution found on $I_{n-i}, i=1, \ldots, n-1$, and, according to (3.4), $V(\pi)=v, \pi \in[0, \varepsilon)$, we obtain a first-order linear differential equation, whose unique solution $\pi \mapsto V(\pi ; \varepsilon, v)$ on $I_{n}$ is

$$
V(\pi ; \varepsilon, v)=c_{n}(\varepsilon) V_{g}(\pi)+V_{p, n}(\pi ; \varepsilon, v), \quad \pi \in I_{n},
$$

where $\pi \mapsto V_{g}(\pi)$ is a general solution given by (3.13), $\pi \mapsto V_{p, n}(\pi ; \varepsilon, v)$ is a particular 
solution, and the constant term $c_{n}(\varepsilon)$ is obtained by imposing a continuity condition over $I_{n-1} \cup I_{n}$ at $\varepsilon_{n-1}$. Making use of the distance function (3.9), the general solution $\pi \mapsto$ $V(\pi ; \varepsilon, v)$ to (3.1) and (3.4) can be expressed as

$$
V(\pi ; \varepsilon, v)= \begin{cases}v, & \pi \in[0, \varepsilon), \\ c_{d(\pi ; \varepsilon)}(\varepsilon) V_{g}(\pi)+V_{p, d(\pi ; \varepsilon)}(\pi ; \varepsilon, v), & \pi \in[\varepsilon, 1) .\end{cases}
$$

We observe that the free-boundary equation (3.1) has no singularity points in the case $p_{0}<p_{1}$.

Remark 3.4. Let $\pi \mapsto f_{1}(\pi)$ and $\pi \mapsto f_{2}(\pi)$ be two maps satisfying (3.1) on $[\varepsilon, 1)$ and $f_{i}(\pi)=v_{i}$ on $[0, \varepsilon], i=1,2$. Then, $\mathbb{L} f_{1}-\mathbb{L} f_{2}=0$ on $[\varepsilon, 1)$ and it is easy to verify that $\mathbb{L} f_{1}-\mathbb{L} f_{2}=\mathbb{L} f$, with $f:=f_{1}-f_{2}$. Using the above arguments, we can straightforwardly prove that $(\mathbb{L} f)(\pi)=0$, for $\pi \in[\varepsilon, 1)$, has as unique solution $f(\pi)=v_{1}-v_{2}, \pi \in[\varepsilon, 1)$. This shows that the maps $\pi \mapsto V\left(\pi ; \varepsilon, v_{i}\right), i=1,2$, given by (3.24), never intersect on $[0,1)$, whenever $v_{1} \neq v_{2}$. This result will be useful in the proof of Theorem 5.1.

\section{Regularity of the boundary point}

In this section we analyze when a given point $B$ in the state space of the posterior probability process $\left(\pi_{t}\right)_{t \geq 0}$ is regular for $(B, 1)$. We recall that $B \in(0,1)$ is regular for $(B, 1)$ if $\mathbb{P}_{\pi=B}(\eta=0)=1$, where $\eta:=\inf \left\{t \geq 0: \pi_{t}>B\right\}$. Generally speaking, $B$ is regular for $(B, 1)$ if $\left(\pi_{t}\right)_{t \geq 0}$ starting at $B$ immediately enters $(B, 1)$. It is well known that the concept of 'regularity' is strictly related to the smooth fit principle (see, e.g. Alili and Kyprianou (2005) and Peskir and Shiryaev (2006, Chapters 3 and 4)).

Proposition 4.1. Let $B \in(0,1)$ be the starting point of $\left(\pi_{t}\right)_{t \geq 0}$. Then,

(i) if $p_{0}>p_{1}$ and $\lambda<\log \left(p_{0} / p_{1}\right), B$ is regular for $(B, 1)$, when $B<\hat{B}$, while $B$ is not regular for $(B, 1)$, when $B \geq \hat{B}$, with $\hat{B}$ given by (3.19);

(ii) if $p_{0}>p_{1}$ and $\lambda \geq \log \left(p_{0} / p_{1}\right)$, $B$ is regular for $(B, 1)$;

(iii) if $p_{0}<p_{1}, B$ is regular for $(B, 1)$.

Proof. For a small $\varepsilon>0$, the relationship $\pi_{t}=\varphi_{t} /\left(1+\varphi_{t}\right)$ implies that

$$
\begin{aligned}
\tau_{B-\varepsilon} & :=\inf \left\{t \geq 0: \pi_{t} \geq B, \pi=B-\varepsilon\right\} \\
& =\inf \left\{t \geq 0: \varphi_{t} \geq \frac{B}{1-B}, \varphi_{0}=\frac{B-\varepsilon}{1-B+\varepsilon}\right\} .
\end{aligned}
$$

According to Sato (1999, Theorem 43.20, p. 323), $X=\left(X_{t}\right)_{t \geq 0}$ from (2.1) satisfies

$$
\mathbb{P}_{\pi}\left[\lim _{t \downarrow 0} t^{-1}\left(a X_{t}+b t\right)=b\right]=1
$$

for any $\pi \in[0,1]$ and any $a, b \in \mathbb{R}$. Hence, for a sufficiently small $t>0$, the quantity $\log \left(q_{1} / q_{0}\right) X_{t}-t\left(\log \left(p_{0} / p_{1}\right)-\lambda\right)$ behaves like $-t\left(\log \left(p_{0} / p_{1}\right)-\lambda\right)$. This fact and (2.5) allow us to rewrite the inequality $\varphi_{t} \geq B /(1-B)$ in (4.1) as

$$
\mathrm{e}^{-t\left(\log \left(p_{0} / p_{1}\right)-\lambda\right)}\left(\frac{B-\varepsilon}{1-B+\varepsilon}+\lambda \int_{0}^{t} \mathrm{e}^{s\left(\log \left(p_{0} / p_{1}\right)-\lambda\right)} \mathrm{d} s\right) \geq \frac{B}{1-B},
$$


for $t>0$ small enough. Denote by

$$
K(\varepsilon):=\log \left(\frac{B /(1-B)-\hat{B} /(1-\hat{B})}{(B-\varepsilon) /(1-B+\varepsilon)-\hat{B} /(1-\hat{B})}\right) .
$$

(i) From (4.2), $B<\hat{B}$, and simple calculations we have

$$
-t\left(\log \left(\frac{p_{0}}{p_{1}}\right)-\lambda\right) \leq K(\varepsilon)(\uparrow 0, \text { as } \varepsilon \downarrow 0) ;
$$

similarly, for $B>\hat{B}$ (with $\varepsilon$ sufficiently small so that $B-\varepsilon>\hat{B}$ ) and $B=\hat{B}$ we have, respectively,

$$
\begin{gathered}
-t\left(\log \left(\frac{p_{0}}{p_{1}}\right)-\lambda\right) \geq K(\varepsilon)(\downarrow 0, \text { as } \varepsilon \downarrow 0) ; \\
\mathrm{e}^{-t\left(\log \left(p_{0} / p_{1}\right)-\lambda\right)} \leq 0
\end{gathered}
$$

Then, (4.3)-(4.5) show that $\tau_{B-\varepsilon} \downarrow 0 \mathbb{P}_{\pi}$-almost surely (a.s.) as $\varepsilon \downarrow 0$ only when $B<\hat{B}$.

(ii) When $\lambda>\log \left(p_{0} / p_{1}\right), \hat{B} /(1-\hat{B})<0$, so that from (4.2) we have

$$
-t\left(\log \left(\frac{p_{0}}{p_{1}}\right)-\lambda\right) \geq K(\varepsilon)(\downarrow 0, \text { as } \varepsilon \downarrow 0) ;
$$

when $\lambda=\log \left(p_{0} / p_{1}\right),(4.2)$ reduces to

$$
\lambda t \geq \frac{B}{1-B}-\frac{B-\varepsilon}{1-B+\varepsilon} .
$$

In both the cases, $\tau_{B-\varepsilon} \downarrow 0 \mathbb{P}_{\pi}$-a.s. as $\varepsilon \downarrow 0$.

(iii) It follows that $\hat{B} /(1-\hat{B})<0$, so (4.2) boils down to (4.6). Then, $\tau_{B-\varepsilon} \downarrow 0 \mathbb{P}_{\pi}$-a.s. as $\varepsilon \downarrow 0$.

The above results can be interpreted in the light of the following conjecture due to Alili and Kyprianou (2005, p.2078): 'It would then be reasonable to work with the assumption that there will be smooth pasting at the boundary if and only if the boundary is regular for the stopping region'. It means that we should expect that the smooth fit principle (3.5) holds at $B^{\star}$ from (2.4) when either $p_{0}>p_{1}$ and $B^{\star}<\hat{B}$, or $p_{0}<p_{1}$.

We note that Proposition 4.1 can be applied in a similar manner to the disorder problems studied by Peskir and Shiryaev (2002) and Gapeev (2005) for formally proving the regularity of the optimal boundary point.

\section{Solution of the optimal stopping problem}

We are now ready to state the main theorem of this paper, which summarizes the previous results.

Theorem 5.1. In the Bayesian disorder problem (2.2) and(2.3) for a negative binomial process, with $p_{0}, p_{1} \in(0,1)$ and $c, \lambda>0$ given and fixed, there exists a unique $B^{\star} \in(0,1)$ such that the stopping time $\tau_{\pi}^{\star}$ defined in (2.4) is optimal in (2.2) and (2.3). We have the following cases. 
(i) If $p_{0}>p_{1}$ and $c>\log \left(p_{0} / p_{1}\right)-\lambda$,

$$
B^{\star}=\widetilde{B}\left(:=\frac{\lambda}{\lambda+c}\right) .
$$

The smooth fit (3.5) holds at $B^{\star}$ and $B^{\star}<\hat{B}$, where $\hat{B}$ is a singularity point of the free-boundary equation (3.1) given in (3.19);

(ii) If $p_{0}>p_{1}$ and $c=\log \left(p_{0} / p_{1}\right)-\lambda, B^{\star}$ is still given by (5.1) and in this case $B^{\star}=\hat{B}$. The smooth fit condition breaks down at $B^{\star}$ and is replaced by (3.20).

(iii) If $p_{0}>p_{1}$ and $c<\log \left(p_{0} / p_{1}\right)-\lambda$, then the smooth fit does not hold at $B^{\star}$ and the latter is the unique solution in $(\hat{B}, 1)$ of the following equation:

$$
c_{d\left(\hat{B} ; B^{\star}\right)}\left(B^{\star}\right)=0,
$$

where the map $B^{\star} \mapsto d\left(\hat{B} ; B^{\star}\right)$ is given through (3.9) and the map $B \mapsto c_{n}(B)$ is expressed by (3.15) and (3.16). In particular, when $c$ satisfies

$$
\frac{q_{0} \log p_{1}\left(\log \left(p_{0} / p_{1}\right)-\lambda\right)}{q_{0} \log p_{1}-\left(\log p_{0}-\lambda\right)\left(p_{1}-p_{0}\right)} \leq c<\log \left(p_{0} / p_{1}\right)-\lambda,
$$

the following equations hold:

$$
B^{\star}=\frac{\lambda\left(\log p_{1}+c\right)}{\lambda \log p_{1}+c \log p_{0}}, \quad V^{\prime}\left(B^{\star}-\right)=\frac{\log p_{0}\left(c+\log p_{1}\right)}{\log p_{1}\left(\lambda-\log p_{0}\right)},
$$

with $B^{\star}$ that, in the case $c=\log \left(p_{0} / p_{1}\right)-\lambda$, reduces to (5.1) again.

In all the above three cases, $\pi \mapsto V(\pi)$ from (2.2) and (2.3) is given by (3.17) and (3.18), with $B$ replaced by $B^{\star}$ and $V(0)=V(0+)$.

(iv) If $p_{0}<p_{1}$, the smooth fit condition holds at $B^{\star}$; in particular, for a fixed $\varepsilon>0$, there exists a unique pair $v_{\varepsilon} \in(0,1)$ and $B_{\varepsilon}^{\star} \in(0,1)$ satisfying

$$
V\left(B_{\varepsilon}^{\star} ; \varepsilon, v_{\varepsilon}\right)=1-B_{\varepsilon}^{\star}, \quad V^{\prime}\left(B_{\varepsilon}^{\star} ; \varepsilon, v_{\varepsilon}\right)=-1,
$$

where the map $\pi \mapsto V(\pi ; \varepsilon, v)$ is given by (3.24). Then,

$$
B^{\star}=\lim _{\varepsilon \downarrow 0} B_{\varepsilon}^{\star}, \quad V(\pi)= \begin{cases}\lim _{\varepsilon \downarrow 0} V\left(\pi ; \varepsilon, v_{\varepsilon}\right), & 0<\pi<B^{\star}, \\ 1-\pi, & B^{\star} \leq \pi \leq 1,\end{cases}
$$

with $V(0)=V(0+)$; see Figure 1 .

Proof. We first note from (2.6) that

$$
\pi_{t}=\pi+\lambda \int_{0}^{t}\left(1-\pi_{s^{-}}\right) \mathrm{d} s+M_{t},
$$

where $\left(M_{t}\right)_{t \geq 0}$ is a $\mathbb{P}_{\pi}$-martingale. Then, standard arguments based on Peskir and Shiryaev (2002), Gapeev (2005) and the optional sampling theorem imply that

$$
\mathbb{E}_{\pi}\left[1-\pi_{\tau}+c \int_{0}^{\tau} \pi_{t} \mathrm{~d} t\right]=1-\pi+(\lambda+c) \mathbb{E}_{\pi}\left[\int_{0}^{\tau}\left(\pi_{t}-\widetilde{B}\right) \mathrm{d} t\right],
$$




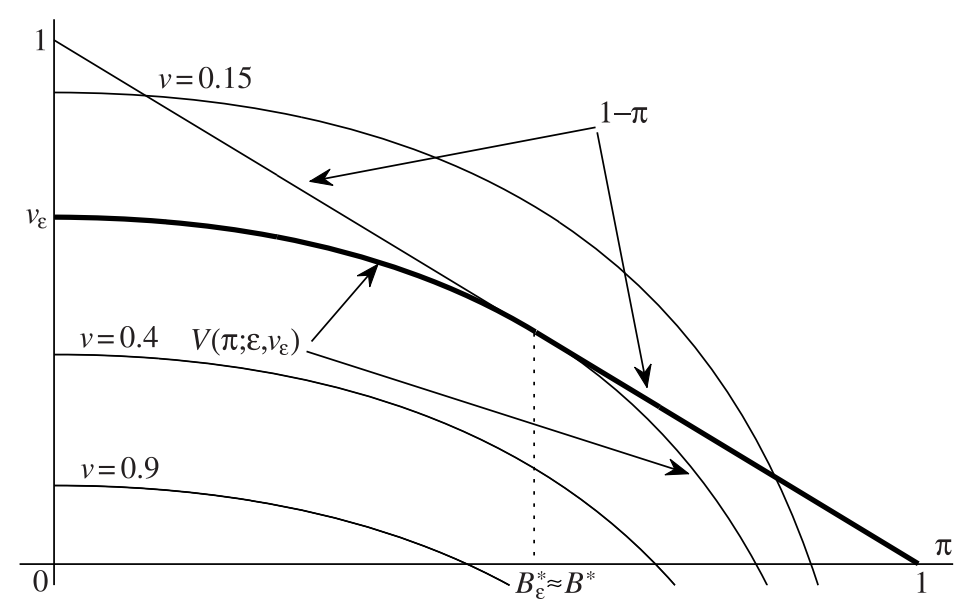

Figure 1: A plot of the map $\pi \mapsto V(\pi)$ (bold line), as defined by (5.6) and coinciding with (2.2) and (2.3). We set $p_{0}=0.3, p_{1}=0.8, \lambda=1, c=1$, and $\varepsilon=0.001$. The unique solution to (5.5) is $v_{\varepsilon}=0.6620$ and $B_{\varepsilon}^{\star}=0.5575$. The other curves are the maps $\pi \mapsto V(\pi ; \varepsilon, v)$, for $v=0.9,0.4$, and 0.15 .

from which we observe that it is never optimal to stop before $\left(\pi_{t}\right)_{t \geq 0}$ reaches $\widetilde{B}$. Hence,

$$
B^{\star} \geq \widetilde{B}
$$

(i) and (ii) According to Gapeev (2005, Lemma 3.1), the fact that $\left(\pi_{t}\right)_{t \geq 0}$ from (2.6) jumps towards 1 whenever $X$ jumps and has drift $(1-\pi) \log \left(p_{0} / p_{1}\right)(\hat{B}-\pi)$, as well as that for $c \geq \log \left(p_{0} / p_{1}\right)-\lambda$ we have $\widetilde{B} \leq \hat{B}$, we see that once $\left(\pi_{t}\right)_{t \geq 0}$ leaves $[0, \hat{B}]$, it will never come back to the propitious set $[0, \widetilde{B})$. Therefore, $B^{\star}=\widetilde{B}$. Remark 3.3 and Proposition 4.1 prove that the smooth fit holds at $B^{\star}$ when $c>\log \left(p_{0} / p_{1}\right)-\lambda$ and breaks down when $c=\log \left(p_{0} / p_{1}\right)-\lambda$.

(iii) When $c<\log \left(p_{0} / p_{1}\right)-\lambda, \hat{B}<\widetilde{B}$, so that from (5.7) it follows that $B^{\star} \in(\hat{B}, 1)$. Since $\pi \mapsto V(\pi)$ is bounded $(V(\pi) \leq 1-\pi)$ and $\hat{B}$ is a singularity point of (3.1), from (3.18) we see that $B^{\star}$ must satisfy (5.2). This is due to the fact that every particular solution $\pi \mapsto V_{p, d\left(\pi ; B^{\star}\right)}\left(\pi ; B^{\star}\right)$ in (3.18) is taken as bounded. The uniqueness of $B^{\star}$ can be proved analogously as in Peskir and Shiryaev (2002, Theorem 4.1, case 3); the fact that the smooth fit does not hold at $B^{\star}$ arises from Proposition 4.1.

We observe that $B^{\star}$ from (5.4) is the unique solution of $c_{1}\left(B^{\star}\right)=0$; the latter, according to the previous reasoning and the definition of $d(\pi ; B)$ from $(3.9)$, characterizes $B^{\star}$ if and only if

$$
0<\frac{\log \left(\left(B^{\star} /\left(1-B^{\star}\right)\right)((1-\hat{B}) / \hat{B})\right)}{\log \left(q_{1} / q_{0}\right)} \leq 1,
$$

which proves (5.3). In this case, (3.12) reduces to (3.14), from which the expression of $V^{\prime}\left(B^{\star}-\right)$ in (5.4) follows.

(iv) We begin by proving that $\pi \mapsto V(\pi ; \varepsilon, v)$ from (3.24) is concave. For a fixed $\varepsilon>0$, let $v_{\varepsilon} \in(0,1)$ and $B_{\varepsilon}^{\star} \in(0,1)$ be such that the map $\pi \mapsto V\left(\pi ; \varepsilon, v_{\varepsilon}\right)$ smoothly hits the map $\pi \mapsto 1-\pi$ at $B_{\varepsilon}^{\star}$. Let $V_{\varepsilon}(\pi):=V\left(\pi ; \varepsilon, v_{\varepsilon}\right), 0 \leq \pi \leq B_{\varepsilon}^{\star}$, and $V_{\varepsilon}(\pi):=1-\pi, B_{\varepsilon}^{\star} \leq \pi \leq 1$. 
Then, it is clear that $\pi \mapsto V_{\varepsilon}(\pi)$ solves the following boundary problem:

$$
\begin{gathered}
\left(\mathbb{L} V_{\varepsilon}\right)(\pi)=-c \pi, \quad \pi \in\left(\varepsilon, B_{\varepsilon}^{\star}\right) ; \\
V_{\varepsilon}(\pi)<v_{\varepsilon} \wedge(1-\pi), \quad \pi \in\left(\varepsilon, B_{\varepsilon}^{\star}\right), \\
V_{\varepsilon}(\pi)=v_{\varepsilon} \wedge(1-\pi), \quad \pi \in[0, \varepsilon] \cup\left[B_{\varepsilon}^{\star}, 1\right], \\
V_{\varepsilon}^{\prime}\left(B_{\varepsilon}^{\star}\right)=-1, \quad(\text { smooth fit }),
\end{gathered}
$$

where $\mathbb{L}$ is given by (2.7) and the smooth fit condition (5.11) should hold according to Proposition 4.1. Define

$$
W(\pi):=\inf _{\tau} \mathbb{E}_{\pi}\left[\left(v_{\varepsilon} \wedge\left(1-\pi_{\tau}\right)\right)+c \int_{0}^{\tau} \pi_{t} \mathrm{~d} t\right],
$$

where the infimum is taken over all the stopping times of $\left(\pi_{t}\right)_{t \geq 0}$. Then, the map $\pi \mapsto V_{\varepsilon}(\pi)$ solving (5.8)-(5.11) equals the map $\pi \mapsto W(\pi)$. In order to prove this claim, first observe that

$$
\left(\mathbb{L} V_{\varepsilon}\right)(\pi) \geq-c \pi, \quad \pi \in[0,1] .
$$

The above inequality is obviously satisfied by construction on the interval $\left(\varepsilon, B_{\varepsilon}^{\star}\right)$. For $\pi \in$ $[0, \varepsilon),\left(\mathbb{L} V_{\varepsilon}\right)(\pi)=0$ because $\mathbb{L} f=0$ if $f(\pi)=k, k \in \mathbb{R}$. It remains to show that (5.13) holds on $\left[B_{\varepsilon}^{\star}, 1\right)$ : it can be proved by adopting the same line of arguments as in Buonaguidi and Muliere (2013a, Theorem 6.1), since $B_{\varepsilon}^{\star}$ is a smooth fit point for the system (5.8)-(5.11); see Figure 2.

Note that $V_{\varepsilon}$ is $C^{1}$ on $[0, \varepsilon) \cup(\varepsilon, 1]$ and $C^{0}$ at $\varepsilon$. Then, since the time spent by the process $\left(\pi_{t}\right)_{t \geq 0}$ at $\varepsilon$ is of Lebesgue measure 0 , Itô's equation can be applied in its standard form to $V_{\varepsilon}$,

$$
V_{\varepsilon}\left(\pi_{t}\right)=V_{\varepsilon}(\pi)+\int_{0}^{t}\left(\mathbb{L} V_{\varepsilon}\right)\left(\pi_{s-}\right) \mathrm{d} s+M_{t}^{\star},
$$

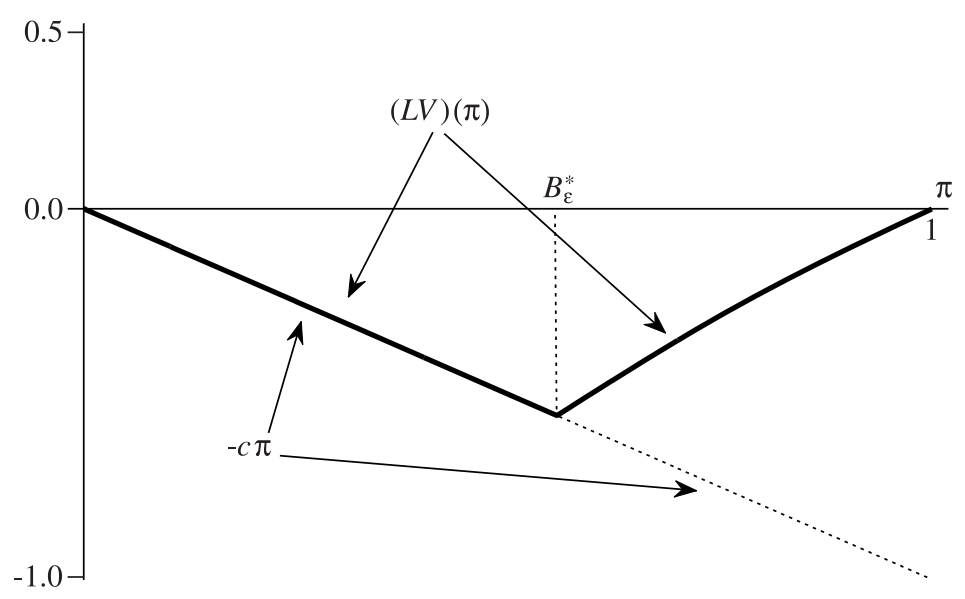

Figure 2: A plot of the map $\pi \mapsto(\mathbb{L} V)(\pi)$, where the infinitesimal operator $\mathbb{L}$ and $V$ are given in (2.7) and (5.6), respectively. From the plot we see that $(\mathbb{L} V)(\pi) \geq-c \pi, \pi \in[0,1]$ : this is a key fact used in the proof of Theorem 5.1, case (iv), for the equivalence between (5.6) and (2.2)-(2.3). The same parameters from Figure 1 are used. 
where $\left(M_{t}^{\star}\right)_{t \geq 0}$, with

$$
M_{t}^{\star}:=\int_{0}^{t} \int_{0}^{1}\left(V_{\varepsilon}\left(\pi_{s-}+y\right)-V_{\varepsilon}\left(\pi_{s-}\right)\right)\left(\mu^{\pi}-v^{\pi}\right)(\mathrm{d} y, \mathrm{~d} s),
$$

is a martingale under $\mathbb{P}_{\pi}, \pi \in[0,1]$, and $\mu^{\pi}$ and $v^{\pi}$ are the measure of jumps and the corresponding compensator of $\left(\pi_{t}\right)_{t \geq 0}$. Let $\tau$ be a stopping time of $\left(\pi_{t}\right)_{t \geq 0}$, such that $\mathbb{E}_{\pi}[\tau]<\infty$. Therefore, by (5.14), the optional sampling theorem, and (5.13), we have

$$
\mathbb{E}_{\pi}\left[V_{\varepsilon}\left(\pi_{\tau}\right)\right]=V_{\varepsilon}(\pi)+\mathbb{E}_{\pi}\left[\int_{0}^{\tau}\left(\mathbb{L} V_{\varepsilon}\right)\left(\pi_{t-}\right) \mathrm{d} t\right] \geq V_{\varepsilon}(\pi)-\mathbb{E}_{\pi}\left[c \int_{0}^{\tau} \pi_{t} \mathrm{~d} t\right],
$$

which, according to (5.9) and (5.10), implies that

$$
V_{\varepsilon}(\pi) \leq \mathbb{E}_{\pi}\left[\left(v_{\varepsilon} \wedge\left(1-\pi_{\tau}\right)\right)+c \int_{0}^{\tau} \pi_{t} \mathrm{~d} t\right]
$$

It proves that $V_{\varepsilon}(\pi) \leq W(\pi)$ for all $\pi \in[0,1]$ and any stopping time with finite expectation. Now, let $\tau_{\varepsilon}:=\inf \left\{t \geq 0: \pi_{t} \notin\left(\varepsilon, B_{\varepsilon}^{\star}\right)\right\} ;$ as $\mathbb{E}_{\pi}\left[\tau_{\varepsilon}\right]<\infty$, from (5.8), the definition of $\tau_{\varepsilon}$, and (5.10), the inequalities in (5.16) and (5.17) become equalities, leading to

$$
V_{\varepsilon}(\pi)=\mathbb{E}_{\pi}\left[\left(v_{\varepsilon} \wedge\left(1-\pi_{\tau_{\varepsilon}}\right)\right)+c \int_{0}^{\tau_{\varepsilon}} \pi_{t} \mathrm{~d} t\right] .
$$

The expressions (5.17) and (5.18) show that $V_{\varepsilon}(\pi)=W(\pi), \pi \in[0,1]$, as well as that $\tau_{\varepsilon}$ is optimal in (5.12). Observe that applying the previous reasoning step by step, we can prove that the maps $\pi \mapsto V(\pi ; \varepsilon, v)$ from (3.24), for different values of $v \in(0,1)$, equal the function (5.12) when $h(v, \pi):=v \wedge(1-\pi)$ is replaced by $g(v, \pi):=v \wedge(\alpha+\beta \pi)$. Since $\pi \mapsto W(\pi)$ from (5.12) is concave, the same holds for $\pi \mapsto V(\pi ; \varepsilon, v)$.

Now, we can prove that $v_{\varepsilon}$ and the smooth fit point $B_{\varepsilon}^{\star}$ are unique. Using (3.21)-(3.23), it is easy to observe that $V(\pi ; \varepsilon, v)>1-\pi$, for $v$ close to 1 and some $\pi>\varepsilon$, and $V(\pi ; \varepsilon, v)<0$, for $v$ close to 0 and some $\pi>\varepsilon$. This fact, the established concavity argument, and Remark 3.4 show that moving $v$ on $(0,1)$ there exists a unique solution $v_{\varepsilon} \in(0,1)$ and $B_{\varepsilon}^{\star} \in(0,1)$ to the system of equations (5.5); see Figure 1.

Finally, the limits in (5.6) follow from the continuity of $\pi \mapsto V(\pi)$ from (2.2) and (2.3), and the normal entrance condition (3.4).

\section{Conclusions}

The quickest detection of a shift in the parameter $p \in(0,1)$ of a negative binomial process has been analyzed. The negative binomial process, as any other compound Poisson process, is of 'finite jump activity' in the sense that it has a finite number of jumps over a finite time interval. Future studies could therefore concern the disorder problem for 'infinite jump activity' processes, which present an infinite number of jumps on any finite time interval. The gamma process and the inverse Gaussian process are two examples. Even though a partial answer to this problem was given by Gapeev (2005), a complete solution seems to be missing.

\section{Acknowledgement}

The authors are grateful to the referee for constructive comments that improved the presentation of the paper. 


\section{References}

Alili, L. ANd Kyprianou, A. E. (2005). Some remarks on first passage of Lévy processes, the American put and pasting principles. Ann. Appl. Prob. 15, 2062-2080.

Anscombe, F. J. (1950). Sampling theory of the negative binomial and logarithmic series distributions. Biometrika 37, 358-382.

Barndorff-Nielsen, O. and Yeo, G. F. (1969). Negative binomial processes. J. Appl. Prob. 6, 633-647.

Bayraktar, E. AND Sezer, S. (2009). Online change detection for a Poisson process with a phase-type change-time prior distribution. Sequential Anal. 28, 218-250.

Buonaguidi, B. ANd Muliere, P. (2013a). Sequential testing problems for Lévy processes. Sequential Anal. 32, 47-70.

BuONAGUIDI, B. AND Muliere, P. (2013b). On the Wald's sequential probability ratio test for Lévy processes. Sequential Anal. 32, 267-287.

Carruthers, P. ANd Minh, D.-V. (1983). A connection between galaxy probabilities in Zwicky clusters counting distributions in particle physics and quantum optics. Phys. Lett. B 131, 116-120.

DaYAniK, S. (2010). Compound Poisson disorder problems with nonlinear detection delay penalty cost functions. Sequential Anal. 29, 193-216.

Dayanik, S. And Sezer, S. O. (2006). Compound Poisson disorder problem. Math. Operat. Res. 31, 649-672.

Gapeev, P. V. (2005). The disorder problem for compound Poisson processes with exponential jumps. Ann. Appl. Prob. 15, 487-499.

Kozubowski, T. J. AND PodgóRski, K. (2009). Distributional properties of the negative binomial Lévy process. Prob. Math. Statist. 29, 43-71.

Mukhopadhyay, N. (2014). Sequential sampling. In International Encyclopedia of Statistical Science, ed. M. Lovric, Springer, Berlin, pp. 1311-1314.

MukHopadhyay, N. AND DE Silva, B. M. (2005). Two-stage estimation of mean in a negative binomial distribution with applications to Mexican bean beetle data. Sequential Anal. 24, 99-137.

Nedelman, J. (1983). A negative binomial model for sampling mosquitoes in a malaria survey. Biometrics 39, 1009-1020.

Peskir, G. AND ShiRyaev, A. N. (2002). Solving the Poisson disorder problem. In Advances in Finance and Stochastics, Springer, Berlin, pp. 295-312.

Peskir, G. AND Shiryaev, A. (2006). Optimal Stopping and Free-Boundary Problems. Birkhäuser, Basel.

Sato, K.-I. (1999). Lévy Processes and Infinitely Divisible Distributions. Cambridge University Press.

Shiryaev, A. N. (1978). Optimal Stopping Rules. Springer, New York.

Vaillant, J. (1991). Negative binomial distributions of individuals and spatio-temporal Cox processes. Scand. J. Statist. 18, 235-248.

Wilson, L. T. AND Room, P. M. (1983). Clumping patterns of fruit and arthropods in cotton, with implications for binomial sampling. Environmental Entomology 12, 50-54.

Zhou, M. ANd CARIN, L. (2013). Negative binomial process count and mixture modeling. IEEE Trans. Pattern Anal. Machine Intelligence, 99, 10.1109/TPAMI.2013.211. 